\title{
Overexpression of SOD1 in Transgenic Rats Protects Vulnerable Neurons Against Ischemic Damage After Global Cerebral Ischemia and Reperfusion
}

\author{
Pak H. Chan, ${ }^{1,3}$ Makoto Kawase, ${ }^{1,3}$ Kensuke Murakami, ${ }^{1}$ Sylvia F. Chen, ${ }^{1,3}$ Yibing Li, ${ }^{1}$ Bernard Calagui, ${ }^{1,3}$ \\ Liza Reola, ${ }^{1,3}$ Elaine Carlson, ${ }^{2}$ and Charles J. Epstein ${ }^{2}$ \\ CNS Injury and Edema Research Center, Departments of ${ }^{1}$ Neurological Surgery and Neurology and ${ }^{2}$ Department of \\ Pediatrics, University of California, School of Medicine, San Francisco, California 94143, and ${ }^{3}$ Departments of \\ Neurosurgery, Neurology and Neurological Sciences, Stanford University School of Medicine, Palo Alto, California 94304
}

Transient global cerebral ischemia resulting from cardiac arrest is known to cause selective death in vulnerable neurons, including hippocampal $\mathrm{CA}_{1}$ pyramidal neurons. It is postulated that oxygen radicals, superoxide in particular, are involved in cell death processes. To test this hypothesis, we first used in situ imaging of superoxide radical distribution by hydroethidine oxidation in vulnerable neurons. We then generated SOD1 transgenic $(\mathrm{Tg})$ rats with a five-fold increase in copper zinc superoxide dismutase activity. The $\mathrm{Tg}$ rats and their non-Tg wild-type littermates were subjected to $10 \mathrm{~min}$ of global ischemia followed by 1 and $3 \mathrm{~d}$ of reperfusion. Neuronal damage, as assessed by cresyl violet staining and DNA fragmentation analysis, was significantly reduced in the hippocampal $\mathrm{CA}_{1}$ region, cortex, striatum, and thalamus in SOD1 $\mathrm{Tg}$ rats at $3 \mathrm{~d}$, as compared with the non-Tg littermates. There were no changes in the hippocampal $\mathrm{CA}_{3}$ subregion and dentate gyrus, resistant areas in both SOD1 Tg and non-Tg rats. Quantitative analysis of the damaged $\mathrm{CA}_{1}$ subregion showed marked neuroprotection against transient global cerebral ischemia in SOD1 Tg rats. These results suggest that superoxide radicals play a role in the delayed ischemic death of hippocampal $\mathrm{CA}_{1}$ neurons. Our data also indicate that SOD1 Tg rats are useful tools for studying the role of oxygen radicals in the pathogenesis of neuronal death after transient global cerebral ischemia.

Key words: superoxide dismutase; transgenic rat; superoxide radicals; transient global cerebral ischemia; delayed neuronal degeneration; DNA fragmentation
Free radicals with unpaired electrons are generally highly reactive molecules that initiate radical chain reactions and damage cellular macromolecules, including proteins, DNA, and lipids, ultimately leading to cell death. Superoxide radicals and other oxygen radicals have been implicated in neuronal cell death in acute CNS injury and in chronic neurodegenerative diseases (Kontos, 1985; Coyle and Puttfarcken, 1993; Chan, 1994). One particular role of oxygen free radicals in brain injury appears to involve reperfusion after cerebral ischemia (Chan, 1996). Reperfusion supplies oxygen to the ischemic region of the brain in which oxygen is being used in mitochondria to generate adenosine triphosphate, and superoxide radicals and $\mathrm{H}_{2} \mathrm{O}_{2}$ are produced as a by-product (Boveris and Chance, 1973). Mitochondria are known to be the site for the production of reactive oxygen species in cultured cortical neurons after exposure to NMDA (Dugan et al., 1995). Although several antioxidant enzymes (including superoxide dismutases [SODs], glutathione peroxidases, and catalase) process these oxygen radicals, when they are overproduced, they generally exceed the capacity of the endogenous antioxidant enzymes, causing oxidative stress or injury of brain cells during reperfusion

Received June 8, 1998; revised July 20, 1998; accepted July 30, 1998.

This work was supported by National Institutes of Health contract, "Transgenic Rat for Stroke Research", NO1-NS-5-2334 and NO1-NS-8-2386 (P.H.C.), and National Institutes of Health Grants NS 14543 (P.H.C.), NS 25372 (P.H.C.), NS 36147 (P.H.C.), and AG 08938 (C.J.E., P.H.C.). P.H.C. is a recipient of the Jacob Javits Neuroscience Investigator Award. We thank Cheryl Christensen for editorial assistance.

Correspondence should be addressed to Dr. Pak H. Chan, Neurosurgical Laboratories, Stanford University, 701B Welch Road, \#148, Palo Alto, CA 94304.

Copyright (C) 1998 Society for Neuroscience $\quad 0270-6474 / 98 / 188292-08 \$ 05.00 / 0$ after an ischemic insult. We have demonstrated that overexpression of cytosolic copper zinc ( $\mathrm{CuZn})$ SOD in transgenic $(\mathrm{Tg})$ mice plays a protective role in several types of brain injury, including cold-injury-induced brain edema (Chan et al., 1991), transient focal cerebral ischemia (Kondo et al., 1997b), traumatic brain injury (Mikawa et al., 1996), and hypoxic and excitotoxic neuronal injury in cultures (Chan et al., 1990; Copin et al., 1996). Although reperfusion after transient global cerebral ischemia resulting from cardiac arrest is known to produce oxygen radicals that damage selective vulnerable neurons, it is not clear whether increased $\mathrm{CuZn}$ SOD activity will provide neuroprotection against this ischemic insult. Mice that overexpress SOD1 would be a perfect choice to address this issue. However, because of the plasticity of the arteries in most mouse strains, SOD1 Tg mice are not well suited for transient global cerebral ischemia, which requires the hypoplasticity of the posterior communicating arteries (Murakami et al., 1997, 1998a).

Despite recent success with the use of $\mathrm{Tg}$ and knock-out mutant mice in elucidating the oxidative mechanisms of brain injury after stroke, there are many obvious advantages to using the rat species for construction of Tg animals. The development of a Tg rat to be applied specifically toward gaining understanding of the underlying mechanisms of oxidative stress in stroke would provide many advantages over the use of Tg or knock-out mice. One of these advantages is the fact that rat global cerebral ischemia models are well established (Pulsinelli et al., 1982; Smith et al., 1984), whereas, application of these models is lacking or scarce in mice. In addition, the cerebrovascular structure, brain anatomy, various physiological parameters, blood hemodynamics, 
and many stroke risk factors have been characterized in rats. The larger size of the animal would permit additional studies, including but not limited to, electrophysiology, microdialysis, studies with systemic cardiovascular variables, regional cerebral blood flow and metabolism, multiorgan physiology, and interaction in both anesthetized and awake animals. For these reasons, as well as for ease of manipulation and performance of physiological measurements, Tg rats that overexpress SOD1 have been made. Using these SOD1 $\mathrm{Tg}$ rats, we now provide evidence that the death of vulnerable hippocampal $\mathrm{CA}_{1}$ neurons is significantly reduced after transient global cerebral ischemia, suggesting a role that superoxide radicals play in delayed ischemic neuronal death.

\section{MATERIALS AND METHODS}

Transgene purification and preparation. A $14 \mathrm{~kb}$ BamHI-Eco RI genomic fragment of the SOD1 gene that contains five exons, and its own promoter, was used for generating Tg rats. The SOD1 genomic DNA was purified from low-melting agarose gel after electrophoresis using EluTip $(\mathrm{S} \& \mathrm{~S})$. The eluted DNA was diluted to $2 \mathrm{ng} / \mu \mathrm{l}$ in $10 \mathrm{~mm}$ Tris, $\mathrm{pH} 7.4$, 2 mM EDTA for injection.

Production of Tg rats. Female Sprague Dawley rats (60-90 gm; Charles River, Holister, CA) were superovulated with $15 \mathrm{U}$ gonadotropin from pregnant mare serum (G4877; Sigma, St. Louis, MO) at 12 P.M. to 2 P.M. followed $48 \mathrm{hr}$ later with $5-15 \mathrm{U}$ human chorionic gonadotropin (CG-5; Sigma), mated to fertile Sprague Dawley males, and checked for "plugs" the following morning. Fertilized eggs were flushed from oviducts using M2 media (M7167; Sigma) at $\sim 2$ P.M. the following day, incubated in hylauronidase (U/ml in M2 media) for $5 \mathrm{~min}$ at room temperature, washed through several changes of M2, placed in a $20 \mu \mathrm{l}$ drop of M16 (M7292; Sigma) overlaid with mineral oil, and incubated at $37^{\circ} \mathrm{C}$ in $7 \% \mathrm{CO}_{2}$.

Fertilized eggs with visible pronuclei were placed in an injection well prepared on a LabTek two-chamber slide with M2 media $(50 \mu 1 \mathrm{M} 2$ media spread to a diameter of $\sim 1 \mathrm{~cm}$ ) and overlaid with mineral oil. The slide was secured to the stage of a Leica DMIRB inverted light microscope equipped with Nomarski DIC optics and two Narishige (Greenvale, NY) three-axis hanging joystick micromanipulators. The DNA was injected using an Eppendorf (Fremont, CA) transjector 5246 and needles fabricated from microcapillaries (TW100F-4; World Precision Instruments, Sarasota, FL) pulled using a Sutter Instruments (Novato, CA) model P-77 micropipette puller. DNA was injected at a concentration of $2 \mathrm{ng} / \mu \mathrm{l}$.

Injected zygotes were transferred the same day (8-10 P.M.) to the right oviduct of $24 \mathrm{hr}$ postcoitus (with vasectomized Sprague Dawley male) "pseudopregnant" female Sprague Dawley rats. Pups were born $24 \mathrm{~d}$ from the day of transfer.

Fluorescence in situ hybridization. The site of transgene integration was determined by fluorescence in situ hybridization (FISH) on metaphase chromosome spreads prepared from blood lymphocytes, as described (Shi et al., 1994). The $12 \mathrm{~kb}$ SOD1 transgene fragment was labeled with digoxigenin-11-dUTP (Boehringer Mannheim, Indianapolis, IN) by nick translation (Enzo Biochem Inc., New York, NY) and detected using fluorescein isothiocyanate-conjugated sheep anti-digoxigenin Fab fragment (Boehringer Mannheim) (Shi et al., 1994). The slides were counterstained with propidium iodide and 4'6-diamidino-2-phenylindole in antifade solution (Oncor, Inc., Gaithersburg, MD) and photographed using ASA 400 Kodak Gold (Eastman Kodak, Rochester, NY) and a Zeiss Photoscope III.

Isoelectric focusing gel electrophoresis. Transgenic animals were identified by expression of the human SOD1 enzyme from red blood cell lysates using horizontal isoelectric focusing gel electrophoresis $(\mathrm{pH}$ 4.5-6.0; Multiphor; Pharmacia Biotech) and staining for SOD1 activity with nitroblue tetrazolium (Sigma) (Epstein et al., 1987). Five microliters of whole blood was lysed in $500 \mu \mathrm{l} 2 \mathrm{~mm}$ EDTA and $0.5 \%$ NP-40. One microliter of lysate was mixed with $10 \mu \mathrm{l} 1 \%$ glycine, loaded onto the gel, and electrophoresed for $2 \mathrm{hr}$ at $2000 \mathrm{~V}$ and $12 \mathrm{~A}$, limited with $0.1 \mathrm{M}$ $\beta$-alanine cathode and $0.1 \mathrm{M}$ glutamic acid in $0.5 \mathrm{M} \mathrm{H}_{3} \mathrm{PO}_{4}$ anode. After electrophoresis, the gel was soaked with gentle mixing in the dark for 10 min in $100 \mathrm{ml}$ of $0.036 \mathrm{M}$ phosphate buffer, $\mathrm{pH} 7.8$, with added $16.1 \mathrm{mg}$ nitroblue tetrazolium, $175 \mu \mathrm{l} N, N, N^{\prime}, N^{\prime}$-tetramethylethylenediamine (Sigma), and $20 \mu 10.5$ m EDTA, pH 8.0, and then removed from the solution, placed on a glass plate, and transferred to a light box to develop for 5-10 min. For analysis of transgene expression in tissues, 2-4 $\mu \mathrm{g}$ of tissue homogenates was electrophoresed as above.

Enzyme activity. The total activity of $\mathrm{CuZn} \mathrm{SOD}$ was determined as previously described (Epstein et al., 1987).

Evaluation of anatomical background. To determine the anatomical background of the ischemia, we evaluated cerebral vasculature in both groups of animals using carbon black injection (Murakami et al., 1998a) with minor modifications. After anesthesia with ketamine $(80 \mathrm{mg} / \mathrm{kg})$ and xylazine $(12 \mathrm{mg} / \mathrm{kg})$, the rats were killed by transcardial perfusion with $200 \mathrm{ml}$ of $10 \mathrm{U} / \mathrm{ml}$ heparin in saline and $300 \mathrm{ml}$ of $3.7 \%$ formaldehyde in $0.1 \mathrm{M}$ PBS. Carbon black, in an equal volume of $20 \%$ gelatin in $\mathrm{H}_{2} \mathrm{O}$ was injected from the ascending aorta. The brain was removed and fixed in $3.7 \%$ formaldehyde in PBS for $24 \mathrm{hr}$. The cerebral vasculature was observed with a dissecting microscope (Stemi 2000C, Zeiss).

Transient global cerebral ischemia. Transient global ischemia was induced by bilateral common carotid artery (CCA) occlusion and bleeding to lower the mean arterial blood pressure to $35-40 \mathrm{mmHg}$, using the previously described method of Smith et al. (1984) with some modifications. Male SOD1 Tg rats (325-540 gm) and control non-Tg littermates were anesthetized with $5 \%$ isoflurane in $70 \% \mathrm{~N}_{2} \mathrm{O}$ and $30 \% \mathrm{O}_{2}$, and maintained during surgery at a level of $1.5-2 \%$ isoflurane in $70 \% \mathrm{~N}_{2} \mathrm{O}$ and $30 \% \mathrm{O}_{2}$ under spontaneous breathing. The rectal temperature was controlled at $37.0 \pm 0.5^{\circ} \mathrm{C}$ during surgery with a feedback-regulated heating pad. The femoral artery was exposed and catheterized with a PE-50 catheter to allow continuous recording of the arterial blood pressure and removal of blood samples for blood gas analysis. The right jugular vein was isolated and cannulated with a 23 gauge butterfly needle for intravenous injection of $500 \mathrm{U} / \mathrm{kg}$ heparin dissolved to $100 \mathrm{U} / \mathrm{ml}$ with $0.9 \%$ saline. After exposing the bilateral CCAs, blood was quickly withdrawn from the jugular vein cannula to achieve a reduction of the mean arterial blood pressure. When the blood pressure reached 40 $\mathrm{mmHg}$, the bilateral CCAs were temporarily occluded with metal clips. The blood pressure was maintained at $35-40 \mathrm{mmHg}$ by additional blood withdrawal and reinfusion. After $10 \mathrm{~min}$, ischemia was terminated by removal of the CCA clips, and the blood was reinfused. Blood flow in the forebrain, measured by laser Doppler flowmetry, dropped to $10 \%$ of the basal level during ischemia and rapidly returned to $100 \%$ after reperfusion. After recovery of the arterial blood pressure, the arterial blood was collected for blood gas analysis. The animals regained consciousness and were maintained in an air-conditioned room at $20^{\circ} \mathrm{C}$ for 1 and $3 \mathrm{~d}$ after reperfusion.

At the end of the recovery period, the rats were deeply anesthetized with methoxyflurane, and the brains were removed and rapidly frozen. Coronal sections of the brains $(20 \mu \mathrm{m})$ were cut with a cryostat and mounted onto slides. The sections were stained with cresyl violet for histological assessment of neuronal cell damage. The brain regions examined were the hippocampus, cortex, thalamus, and striatum. The histopathological damage was measured using a neuropathological scoring system of $0-6$, in which $0=$ no damage, $1=0-10 \%, 2=10-25 \%$, $3=25-50 \%, 4=50-75 \%, 5=75-100 \%$, and $6=$ complete neuronal death (McBean et al., 1995). Scores for SOD1 Tg rats were compared with those for the non- $\mathrm{Tg}$ littermates by means of a nonparametric analysis using the Mann-Whitney $U$ test. The lesion areas of the hippocampal $\mathrm{CA}_{1}$ subregion were quantified using the image analysis system of Swanson et al. (1990) with some modification. The present method was created to evaluate infarct size in focal ischemia by measuring areas of the stained sections that had optical densities exceeding a threshold value and was applied to measure the neuronal damage in the $\mathrm{CA}_{1}$ subregion after global ischemia without sampling error and observer bias. Fresh frozen brains were sectioned with a cryostat into a $20 \mu \mathrm{m}$ thickness from the anterior side to the posterior side at $1 \mathrm{~mm}$ intervals, consecutively. The sections were mounted on slide glass and stained with cresyl violet using standard histological criteria. The stained images were scanned by a Color One scanner (Apple Computer, Cupertino, CA). In each hippocampal section the lengths of the unstained and total $\mathrm{CA}_{1}$ subregions were measured by the NIH image program. The area of the damaged and total $\mathrm{CA}_{1}$ pyramidal cell layer was calculated by integrating the length of the damaged and total $\mathrm{CA}_{1}$ pyramidal cell layers by the distance. The ratio of $\mathrm{CA}_{1}$ damage was calculated as (area of $\mathrm{CA}_{1}$ damage/area of total $\mathrm{CA}_{1}$ subregion) $\times 100 \%$. The hemisphere area at the posterior commissure level in the coronal sections was also measured using the same method to evaluate brain swelling and edema. Results were expressed as mean \pm SE. The statistical significance of differences between the SOD1 $\mathrm{Tg}$ rat and non-Tg littermates was evaluated by Fisher's protected least 
significant difference test followed by the nonparametric $t$ test. Significance between groups was assigned at a level of $<5 \%$ probability.

In situ detection of superoxide anion $\left(\mathrm{O}_{2}^{-}\right)$production. The spatial production of $\mathrm{O}_{2}^{-}$during cerebral ischemia was investigated by the in situ detection of oxidized hydroethidine (HEt) method as previously described (Kondo et al., 1997a; Murakami et al., 1998b) with minor modifications. HEt (Molecular Probes, Eugene, OR) is taken up by living cells and oxidized to a red fluorescent dye, ethidium, specifically by $\mathrm{O}_{2}^{-}$, but not by other reactive oxygen species in the cells (Bindokas et al., 1996). The rats were anesthetized with $2 \%$ isoflurane in $30 \% \mathrm{O}_{2}$ and $70 \% \mathrm{~N}_{2} \mathrm{O}$. The HEt solution $(1 \mathrm{ml} ; 1 \mathrm{mg} / \mathrm{ml}$ in $1 \%$ dimethylsulfoxide with PBS) was administered intravenously $1 \mathrm{hr}$ before killing. The rats were killed at $1 \mathrm{hr}$ and 1 and $3 \mathrm{~d}$ after ischemia by transcardial perfusion with $10 \mathrm{U} / \mathrm{ml}$ heparin in saline and $3.7 \%$ formaldehyde. After postfixation, the brains were cut into slices of $50 \mu \mathrm{m}$ thickness at the level of the anterior commissure and the hippocampus using a vibratome and placed on glass slides. These sections were analyzed under fluorescent light (HBO $100 \mathrm{~W} / 2$, Zeiss), and fluorescence was assessed at excitation $=510-550 \mathrm{~nm}$ and emission $>580 \mathrm{~nm}$ for detection of ethidium. Photomicrographs of the hippocampus and the cortex were taken, and the intensity and expression patterns of the oxidized HEt were compared with control nonischemic brains and between each period after $10 \mathrm{~min}$ of global ischemia.

In situ detection of cells with fragmented DNA. DNA fragmentation after global ischemia was determined by the terminal deoxynucleotidyl transferase-mediated uridine $5^{\prime}$-triphosphate-biotin nick end labeling (TUNEL) method in the brains of Tg and control rats. Frozen hippocampal brain sections were stained as previously described (Murakami et al., 1998b) with minor modifications. In brief, frozen brain sections were fixed with $3.7 \%$ formaldehyde. After endogenous peroxidase was inactivated with $0.3 \% \mathrm{H}_{2} \mathrm{O}_{2}$ for $30 \mathrm{~min}$, the sections were immersed in terminal deoxynucleotidyl transferase (TdT) buffer (Life Technologies, Gaithersburg, MD) and incubated with TdT and biotin-16-uridine-5'triphosphate (Boehringer Mannheim). After blocking with 2\% bovine serum albumin in PBS, the sections were incubated with avidin-biotinhorseradish peroxidase (ABC kit; Vector Laboratories, Burlingame, CA) and visualized with $3 \mathrm{~mm}$ 3,3'-diaminobenzidine tetrahydrochloride and $18 \mathrm{~mm}$ hydrogen peroxide in PBS. The slides were counterstained with methyl green and mounted.

TUNEL-positive cells were quantified with a light microscope by a blinded investigator. The total number of cells and the number of TUNEL-positive cells within a grid were counted using high-powered magnification $(400 \times)$. The ratio of the number of TUNEL-positive neurons to the total injured neurons was calculated and expressed as percent of the TUNEL-positive cells in each group.

\section{RESULTS}

\section{Characterization of SOD1 $\mathrm{Tg}$ rats}

SOD1 Tg rats [number 66, University of California San Francisco (UCSF)] have been successfully produced. In this strain, the human SOD1 transgene was incorporated into a single chromosome as shown by FISH (Fig. 1). Because the human and rat SOD proteins comigrate in a nondenaturing gel system (Epstein et al., 1987), we developed a new nondenaturing isoelectric focusing gel electrophoresis (IFGE) method to separate these two proteins, thereby permitting semiquantitative assay of these two enzymes. The human homodimer and the heterodimer composed of human and rat segments were clearly demonstrated in various brain regions, including the hippocampus, brainstem, cerebellum, spinal cord, pituitary gland, cerebral cortex, and striatum, as well as in heart tissue in the IFGE gel (Fig. 2). Because of the high expression of the human transgene relative to the endogenous rat genes, the rat $\mathrm{CuZn}$ SOD homodimer was rarely visualized. Total $\mathrm{CuZn}$ SOD activity ranged from $8.2-27.2 \mathrm{U} / \mathrm{mg}$ in various brain regions as compared with the range of $2.5-5.3 \mathrm{U} / \mathrm{mg}$ in non-Tg rat counterparts (Table 1). The CuZn SOD activity, therefore, increased $\sim 5.2-6.2$-fold in the $\mathrm{Tg}$ rats.

The cerebral vasculature was analyzed to confirm that both anatomical backgrounds were the same in SOD1 Tg rats and non-Tg littermates. Cerebral vasculature was determined by car-

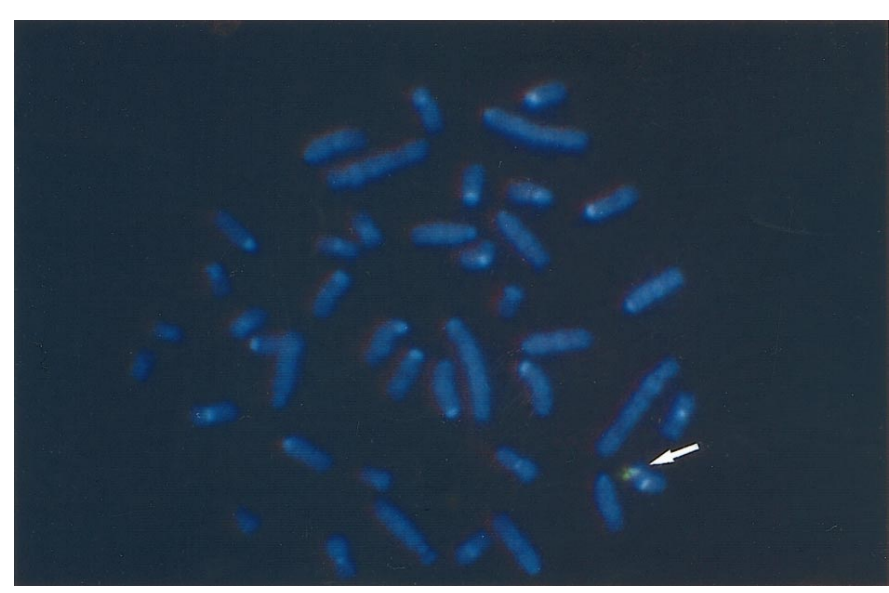

Figure 1. Incorporation of human $\mathrm{CuZn} \mathrm{SOD} \mathrm{(SOD1)} \mathrm{transgene} \mathrm{into} \mathrm{a}$ single rat chromosome detected by fluorescence in situ hybridization. Note the spots of bright fluorescence located in one of the rat chromosomes.

bon black injection (Fig. 3). The method used herein allowed us to determine the structure of major blood vessels (Fig. $3 A, B$ ) in the brain and the posterior communicating artery (Fig. $3 C, D$ ) that influence the ischemic status in global ischemia. There was no remarkable difference between SOD1 $\mathrm{Tg}$ rats and non-Tg littermates.

\section{Production of $\mathrm{O}_{2}^{-}$during global cerebral ischemia}

Before the use of SOD1 Tg rats for transient global cerebral ischemia studies, we first wanted to verify the increased production of $\mathrm{O}_{2}^{-}$in the brains of animals after transient cerebral ischemia, especially in the areas with vulnerable neurons (i.e., hippocampal $\mathrm{CA}_{1}$ subregion). Therefore, production of $\mathrm{O}_{2}^{-}$was determined using $\mathrm{HEt}$ in the cortex and hippocampal $\mathrm{CA}_{1}$ and $\mathrm{CA}_{3}$ subregions at $1 \mathrm{hr}$ and 1 and $3 \mathrm{~d}$ after $10 \mathrm{~min}$ global ischemia. As previously observed by Kondo et al. (1997a), $\mathrm{O}_{2}^{-}$production was shown by oxidized HEt signals as small particles in the cytosol, suggesting possible mitochondrial production of $\mathrm{O}_{2}^{-}$under normal physiological conditions (Fig. 4A-C).

At $1 \mathrm{hr}$ after ischemia and reperfusion, the increase in the diff use cytosolic signal of oxidized HEt was markedly observed in cortical cells (Fig. 4D). In the hippocampus the diffuse cytosolic signal of oxidized HEt was also observed, but the intensity was similar to that of the preischemic level (Fig. 4E,F). At $1 \mathrm{~d}$ after ischemia, the diff use cytosolic signals in cortical cells returned to the preischemic level, and, except for the endothelial cells (Fig. $4 G)$, the marked expression of the signal was present in the hippocampal $\mathrm{CA}_{1}$ subregion (Fig. $4 H$ ). The cytosol was filled with diffuse oxidized HEt fluorescence in these cells. However, signals were not observed in the $\mathrm{CA}_{3}$ subregion (Fig. 4I). The oxidized HEt signals decreased at $3 \mathrm{~d}$ after ischemia (Fig. $4 J-L$ ). The observation of increased production of $\mathrm{O}_{2}^{-}$in vulnerable brain regions prompted us to proceed to the use of SOD1 Tg rats for the transient global cerebral ischemia studies.

\section{Delayed neuronal injury after transient global cerebral ischemia}

There were no marked differences in mortality between SOD1 Tg rats $(10 \%)$ and non-Tg littermates $(7.7 \%)$ within $72 \mathrm{hr}$ after global ischemia and reperfusion. No significant difference was observed in any physiological parameters between the two groups (Table 2). As shown in Figure 5, the ischemic damage can be 


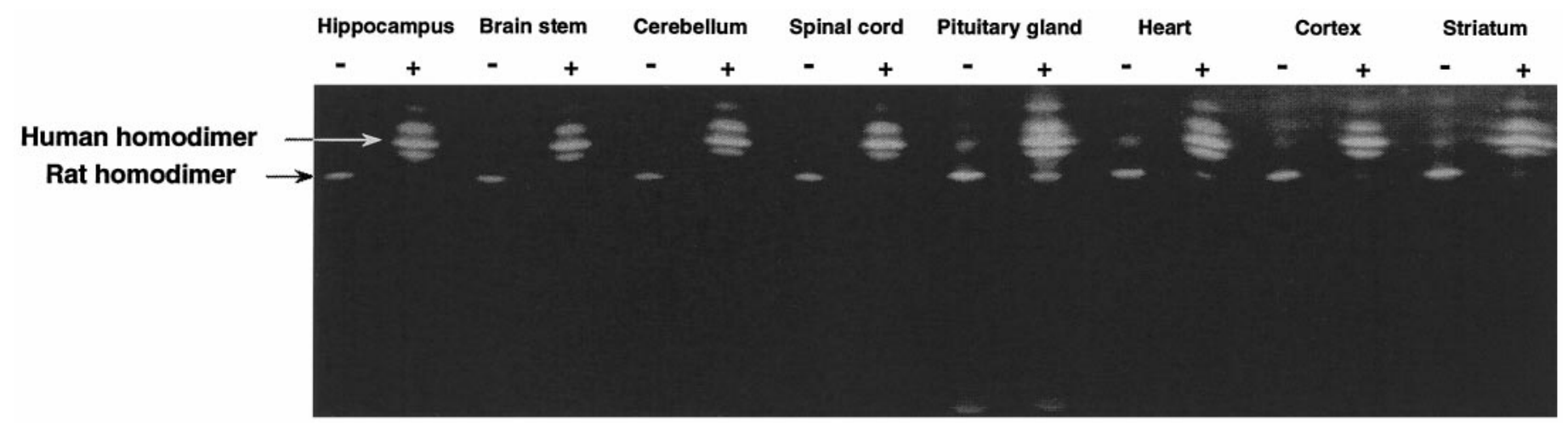

Figure 2. Expression of human CuZn SOD in various brain regions and heart tissue in SOD1 Tg rat. The tissue homogenates from various brain regions and from heart tissue were subjected to isoelectric focusing gel electrophoresis. The short arrow indicates the homodimer of rat CuZn SOD. The long arrow indicates the homodimer of the human CuZn SOD. The band between the rat and human CuZn SOD homodimers is the rat/human heterodimer.

\begin{tabular}{lllll}
\hline \multicolumn{3}{l}{ Table 1. SOD activity in regions of the rat brain } & \\
\cline { 2 - 2 } Region & $\mathrm{Non}-\mathrm{Tg}$ & & $\mathrm{Tg}$ & \\
\cline { 2 - 2 } Cortex & $2.5 \pm 0.2$ & & $14.3 \pm 1$ & 5.8 \\
Striatum & $2.8 \pm 0.3$ & & $18.2 \pm 1.9$ & 5.5 \\
Hippocampus & $4.5 \pm 0.5$ & & $23.5 \pm 3.7$ & 5.2 \\
Brainstem & $5.2 \pm 0.4$ & & $27 \pm 2.6$ & 5.3 \\
Cerebellum & $4.4 \pm 0.6$ & & $27.2 \pm 3.9$ & 6.2 \\
Spinal cord & $4.5 \pm 0.5$ & & $23.5 \pm 3.7$ & 5.2 \\
\hline
\end{tabular}

defined by cresyl violet staining. Ischemic change and severe brain swelling at $3 \mathrm{~d}$ after global ischemia were seen in the non-Tg littermates (Fig. $5 A$ ). In contrast, this damage was reduced in the SOD1 Tg rats. Additionally, the quantitative analysis of the area of hemisphere at the level of the posterior commissure clearly showed that the swelling was markedly reduced in SOD1 Tg rats $\left(57.3 \pm 0.8 \mathrm{~mm}^{2}\right)$ compared with non-Tg littermates (62.2 \pm 1.2 ; $p<0.01)$. The pyramidal neurons were not clearly stained in the non-Tg littermates, especially in the hippocampal $\mathrm{CA}_{1}$ subregion (Fig. $5 C, E$ ). However, many $\mathrm{CA}_{1}$ neurons in the SOD1 $\mathrm{Tg}$ rats were clearly observed as being without degeneration (Fig. $5 D, F$ ). The grading data of the neuronal damage were significantly reduced in the cortex of SOD1 $\mathrm{Tg}$ rats compared with non- $\mathrm{Tg}$ littermates, striatum, thalamus, and in the hippocampal $\mathrm{CA}_{1}$ subregion, whereas no significant changes were seen after ischemia and reperfusion in the dentate gyrus (Fig. 6A). There was no significant reduction of injuries in the $\mathrm{CA}_{3}$ subregion, although a trend toward significance was observed. This was because damage to the $\mathrm{CA}_{3}$ subregion did occur in the SOD1 Tg rats. The quantitative analysis of the damaged area of the $\mathrm{CA}_{1}$ subregion showed a markedly protective effect against global ischemia in SOD1 Tg rats compared with non-Tg littermates $3 \mathrm{~d}$ after ischemia (Fig. 6B). However, no significant differences were detected $1 \mathrm{~d}$ after reperfusion.

\section{DNA fragmentation}

To determine the role of CuZn SOD in DNA fragmentation in delayed neuronal damage, we used in situ TUNEL staining to evaluate the contribution of DNA fragmentation to neuronal damage after global ischemia. We did not observe DNAfragmented nuclei labeled by TUNEL staining at $1 \mathrm{~d}$ after global ischemia (Fig. 7A,B). At $3 \mathrm{~d}$ after global ischemia, the TUNEL-
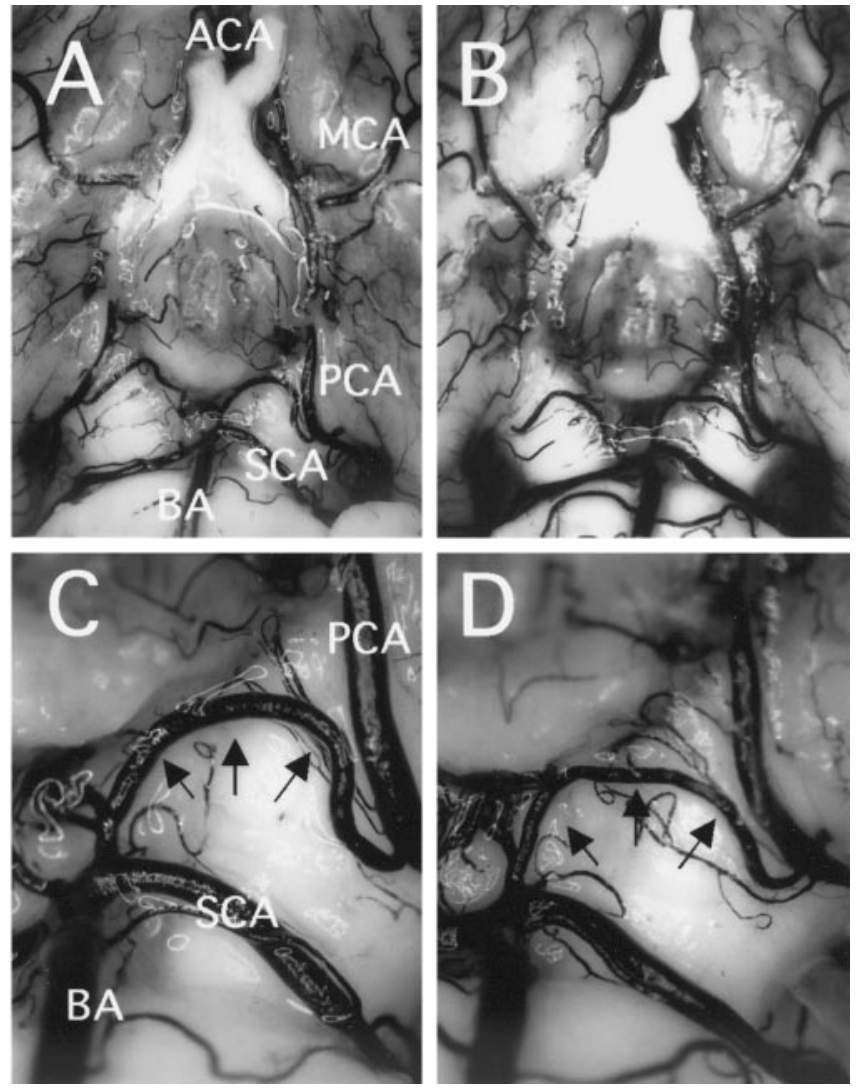

Figure 3. Photographs showing similarity in cerebral vasculature in non-Tg littermates (left) and SOD1 Tg rats (right). The major blood vessels involved in the circle of Willis were almost the same in both animals $(A, B)$. The plasticity of the posterior communicating artery (arrows) that influences the outcome of hippocampal injury after global ischemia was similar between the two animals $(C, D)$. ACA, Anterior cerebral artery; MCA, middle cerebral artery; PCA, posterior cerebral artery; SCA, superior cerebellar artery; BA, basilar artery.

positive cells were restricted to the hippocampal $\mathrm{CA}_{1}$ subregion (Fig. $7 C, D$ ), and the number of TUNEL-positive cells observed in the $\mathrm{CA}_{1}$ subregion of the non-Tg littermates (Fig. $7 C$ ) was much greater than in the SOD1 Tg rats (Fig. $7 D$ ).

To determine the temporal pattern of DNA fragmentation after global ischemia, we counted TUNEL-positive cells in the hippocampal $\mathrm{CA}_{1}$ subregion at 1 and $3 \mathrm{~d}$ (Fig. $7 E$ ). Quantitative 
Figure 4. Superoxide radical imaging in ischemic brain. Representative photographs showing the production of $\mathrm{O}_{2}^{-}$by the presence of oxidized HEt in cortex (left), hippocampal $\mathrm{CA}_{1}$ (middle), and $\mathrm{CA}_{3}$ (right) subregions. As previously observed by Kondo et al. (1997a), O $\mathrm{O}_{2}^{-}$production was demonstrated under normal physiological conditions by oxidized HEt signals appearing as small particles in the cytosol, suggesting possible mitochondrial production of $\mathrm{O}_{2}^{-}(A-C)$. At $1 \mathrm{hr}$ after ischemia and reperfusion, the diffuse cytosolic oxidized HEt signal was observed in cortical cells $(D)$. Although a high background could be observed, the cytosolic signals were not increased in the hippocampal $\mathrm{CA}_{1}(E)$ and $\mathrm{CA}_{3}(F)$ subregions. At $1 \mathrm{~d}$ after ischemia, the diff use cytosolic signals attenuated to preischemic levels in cortical cells except for endothelial cells $(G)$. However, the marked diff use cytosolic signal remained in the hippocampal $\mathrm{CA}_{1}$ subregion $(H)$ but not in the $\mathrm{CA}_{3}$ subregion $(I)$. The diff use signal was not found at $3 \mathrm{~d}$ after ischemia $(J-L)$. Scale bar, $200 \mu \mathrm{m}$ (lower magnification); $20 \mu \mathrm{m}$ (higher magnification).
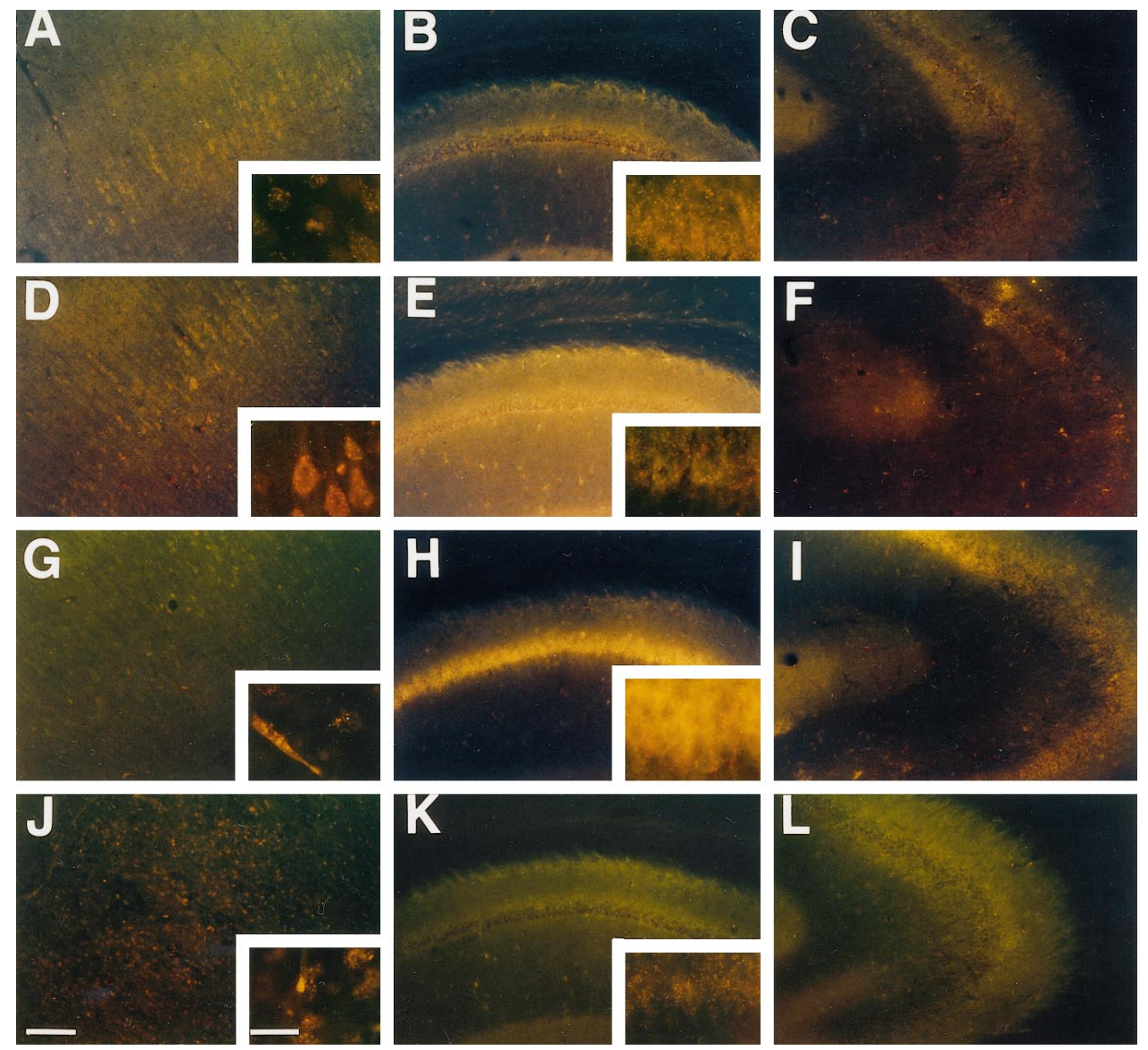

\section{Table 2. Physiological parameters}

\begin{tabular}{llllllll} 
& \multicolumn{2}{l}{ Arterial blood gas analysis } & & \multicolumn{3}{l}{ Mean arterial blood pressure $(\mathrm{mmHg})$} \\
\cline { 2 - 4 } Genotype & $\mathrm{pH}$ & $\mathrm{pO}_{2}$ (torr) & $\mathrm{pCO}_{2}$ (torr) & & Preischemia & Ischemia & Postischemia \\
\hline SOD1 $\mathrm{Tg}$ & $7.39 \pm 0.02$ & $118.8 \pm 11.9$ & $44.9 \pm 1.6$ & & $104.0 \pm 3.1$ & $36.4 \pm 0.4$ & $125.0 \pm 10.0$ \\
Non-Tg & $7.34 \pm 0.03$ & $116.4 \pm 10.4$ & $50.1 \pm 3.7$ & & $102.6 \pm 2.2$ & $36.8 \pm 0.5$ & $128.8 \pm 4.1$ \\
\hline
\end{tabular}

All data are expressed as mean \pm SE. The arterial samples were collected just after reperfusion. No significant difference was observed between SOD1 Tg rats and non-Tg littermates $(n=5, p>0.05)$.

analysis showed that the TUNEL-positive neurons in the hippocampal $\mathrm{CA}_{1}$ subregion did not appear $1 \mathrm{~d}$ after global ischemia and reperfusion. Although DNA-fragmented cells were observed $3 \mathrm{~d}$ after global ischemia in both groups, DNA fragmentation damage was significantly ameliorated in the $\mathrm{Tg}$ rats compared with the non-Tg littermates $(n=6, p<0.03$, Fisher's protected least significant difference test, followed by the nonparametric $t$ test). However, the same level of TUNEL-stained cells was observed between the $\mathrm{Tg}$ and non-Tg rats $1 \mathrm{~d}$ after reperfusion $(n=4)$.

\section{DISCUSSION}

\section{Early superoxide radical formation in hippocampal $\mathbf{C A}_{1}$ subregion after transient global cerebral ischemia in rats}

It is well established that transient global cerebral ischemia manifested by cardiac arrest causes selective neuronal death in vulnerable regions, such as hippocampal $\mathrm{CA}_{1}$ pyramidal cells, Purkinje cells of the cerebellum, and neurons in the third to fifth layers of the cerebral cortex (Kirino, 1982; Pulsinelli et al., 1982). The cell death mechanisms of these vulnerable neurons after transient cerebral ischemia have been extensively studied and are attributed to many factors, including glutamate neurotoxicity, calcium, expression of various genes, mitochondrial dysfunction, and oxygen free radicals (Abe et al., 1995; Ito et al., 1997). The oxygen free radical hypothesis is especially attractive because of the phenomenon of oxygen radical production, superoxide radicals in particular, that is associated with reperfusion injury (McCord, 1985; Chan, 1996). However, it is technically difficult to prove or disprove this hypothesis because of the lack of a quantitative method for oxygen radical measurement in the ischemic brain and the lack of a direct correlation between the increased antioxidant levels and their neuroprotection in experimental animal models of transient global cerebral ischemia (Chan et al., 1996).

To address the first issue, we have developed an in situ imaging method for superoxide radical measurement (Kondo et al., 1997a; 

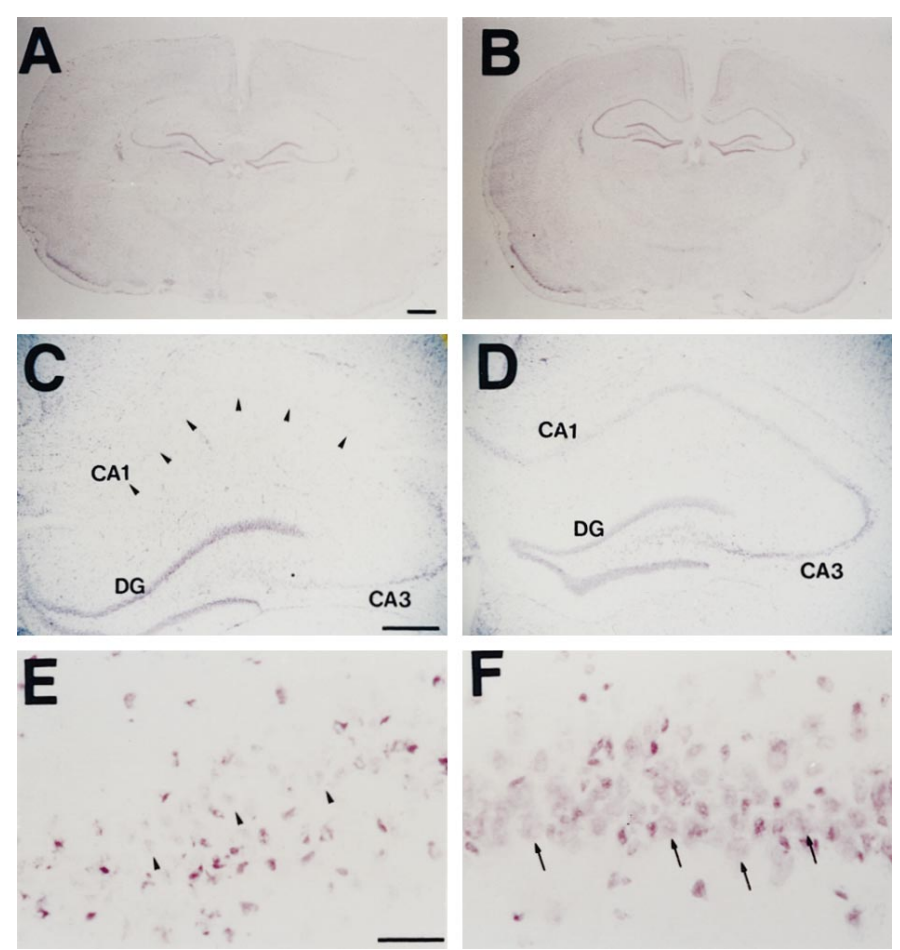

Figure 5. Microscopic photographs showing neuronal damage stained with cresyl violet in SOD1 Tg rats and non-Tg littermates $3 \mathrm{~d}$ after transient global ischemia. More severe edema was observed in non-Tg littermates $(A)$ than in $\mathrm{Tg}$ rats $(B)$. The $\mathrm{CA}_{1}$ pyramidal cell layer in non-Tg littermates was weakly stained by cresyl violet ( $C$, arrowheads), and the nuclei of $\mathrm{CA}_{1}$ neurons were shrunken (E, arrowheads) compared with those of SOD1 Tg rats $(D, F$, arrows). Scale bars: $A, B, 1 \mathrm{~mm} ; C, D$, $500 \mu \mathrm{m} ; E, F, 10 \mu \mathrm{m}$.

Murakami et al., 1998b). This method is based on the selective oxidation of HEt by superoxide radicals (Bindokas et al., 1996). At $1 \mathrm{hr}$ after $10 \mathrm{~min}$ of ischemia and reperfusion, we observed diffuse cytosolic expression of oxidized HEt signals in cortical cells (Fig. 4D). These oxidized HEt signals were significantly increased in vulnerable hippocampal $\mathrm{CA}_{1}$ neurons, whereas they were not observed in the ischemia-resistant $\mathrm{CA}_{3}$ neurons. The increased level of superoxide radicals precedes the occurrence of the majority of neuronal cell deaths and DNA fragmentation detected $3 \mathrm{~d}$ after reperfusion in rats (Figs. 5, 7). These data prompted our further investigation of the role of SOD1 in $\mathrm{CA}_{1}$ neuroprotection after transient global cerebral ischemia.

\section{The making of SOD1 $\mathrm{Tg}$ rats for transient global cerebral ischemia}

$\mathrm{CuZn}$ SOD has been extensively used in attempts to reduce brain injury induced by ischemia and reperfusion. Various degrees of success and failure were obtained in neuroprotection when exogenous CuZn SOD was used (Chan et al., 1993). However, the neuroprotective role of $\mathrm{CuZn} \mathrm{SOD}$ in transient focal cerebral ischemia was found in $\mathrm{Tg}$ mice overexpressing SOD1 activity (Kinouchi et al., 1991; Yang et al., 1994). Conversely, increased neuronal injury was observed in knock-out mutant mice deficient in SOD1 activity (Kondo et al., 1997b). However, the extremely high mortality of mice that were subjected to global cerebral ischemia and the variability in the anatomy of the cerebrovasculature and in the genetic background of the $\mathrm{Tg}$ and knock-out mutant mice prompted us to produce SOD1 $\mathrm{Tg}$ rats. Using
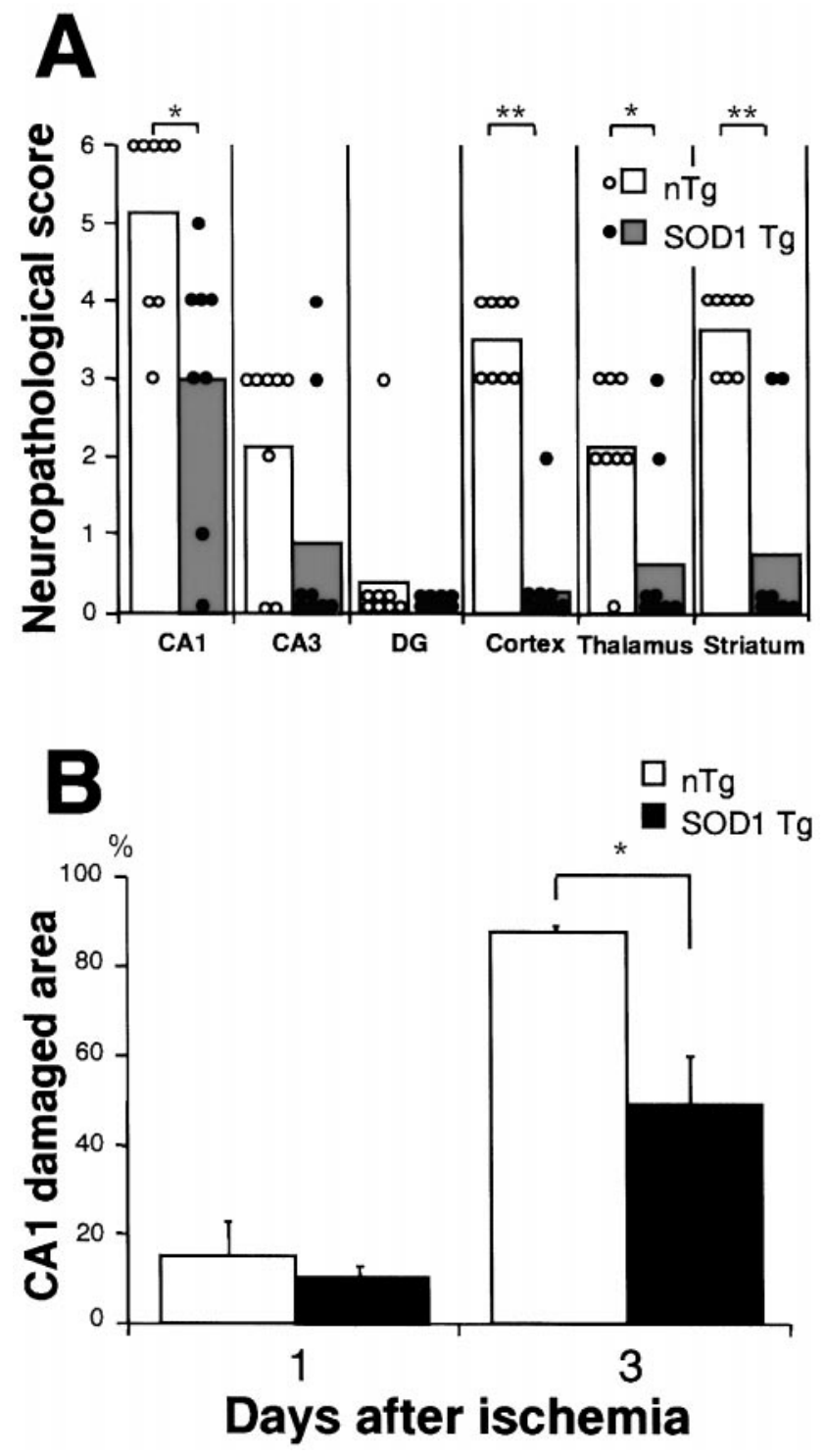

Figure 6. Neuronal injury after transient global cerebral ischemia in rats. $A$, Data showing the scatterplot of the neuropathological scores for a variety of brain areas for SOD1 Tg rats (closed circles, $n=8$ ) and non-Tg littermates (open circles, $n=8$ ) after global ischemia of $10 \mathrm{~min}$ and reperfusion of $3 \mathrm{~d}$. Each column shows the mean score of injury of each group. ${ }^{*} p<0.03,{ }^{*} p<0.01$, significantly different from non-Tg littermates (Mann-Whitney $U$ test). $B$, The quantitative analysis of the damaged area of the $\mathrm{CA}_{1}$ subregion at 1 and $3 \mathrm{~d}$ after $10 \mathrm{~min}$ of global ischemia, and reperfusion showed a marked increase in the size of the damaged area between 1 and $3 \mathrm{~d}$ in both groups. The protective effect of $\mathrm{CuZn}$ SOD against global ischemia was significant in SOD1 Tg rats $(48.4 \pm 11.7 \%$; closed bar) compared with non-Tg littermates $(87.3 \pm$ $1.7 \%$; open bar; $n=6, p<0.01$ ) at $3 \mathrm{~d}$ after ischemia. However, no significant differences were observed $1 \mathrm{~d}(n=4)$ after ischemia. Values are mean \pm SE (Fisher's protected least significant difference test followed by nonparametric $t$ test).

standard pronuclear DNA injection in rat embryos, we have succeeded in making several strains of transgenic rats that overexpress human $\mathrm{CuZn}$ SOD activity in the brain and in other systemic organs (E. Carlson, C. Epstein, and P. H. Chan, unpublished data). A heterozygous Tg strain (number 66 UCSF) that overexpresses $\mathrm{CuZn}$ SOD has been fully characterized by FISH (Fig. 1), IFGE (Fig. 2), and by direct enzymatic assay (Table 1). 


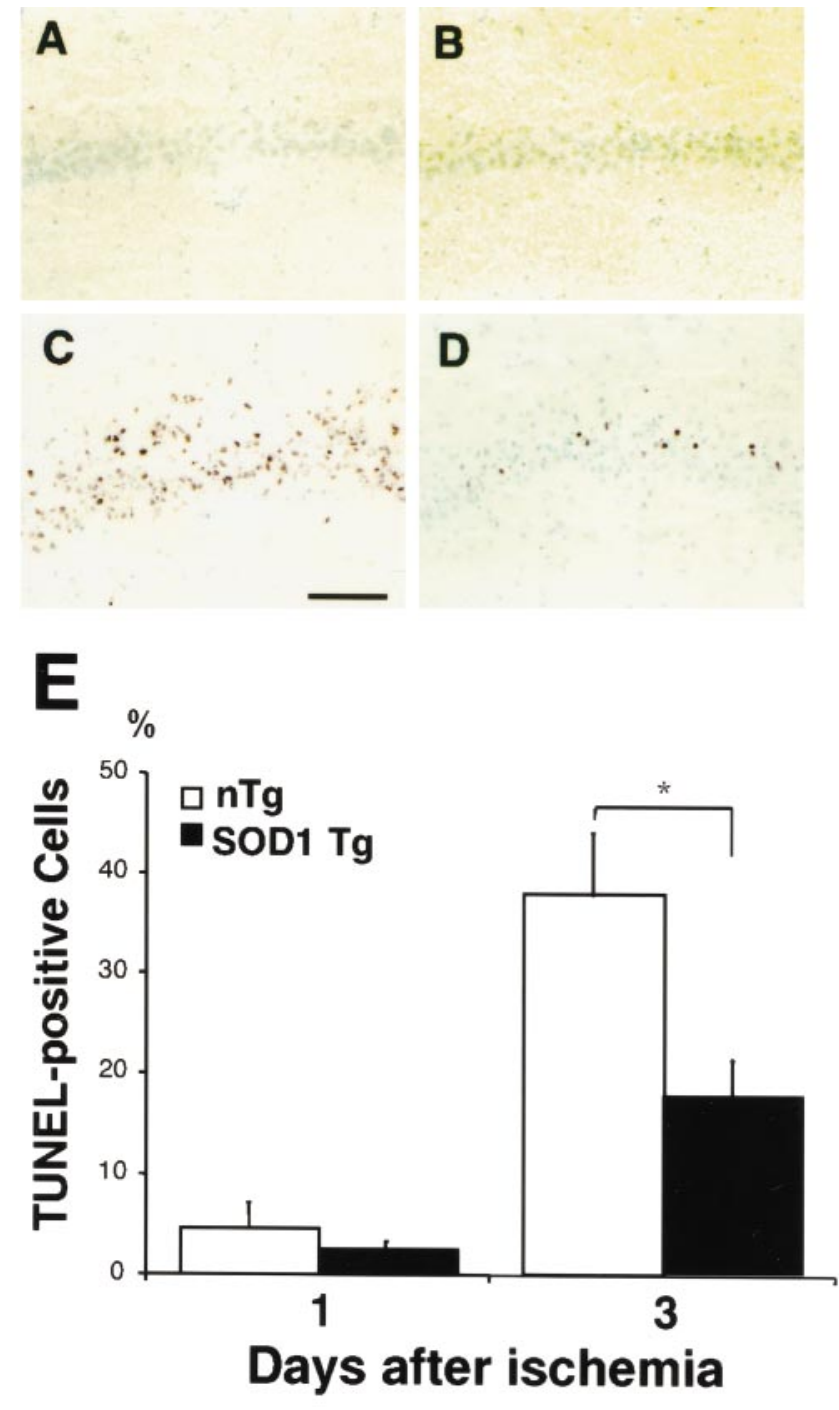

Figure 7. DNA fragmentation. Representative photographs showing DNA fragmentation by TUNEL staining at $1 \mathrm{~d}(A, B)$ and $3 \mathrm{~d}(C, D)$ after $10 \mathrm{~min}$ of global ischemia and reperfusion. The DNA-fragmented cells labeled by TUNEL staining were restricted to the hippocampal $\mathrm{CA}_{1}$ subregion at $3 \mathrm{~d}$ after global ischemia, but not at $1 \mathrm{~d}$ after global ischemia. A marked number of TUNEL-positive cells was observed in the $\mathrm{CA}_{1}$ subregion in non-Tg littermates $(C)$, compared with SOD1 Tg rats $(D)$. Scale bar, $500 \mu \mathrm{m}$. Quantitative analysis showed the ratio of TUNELpositive neurons in the hippocampal $\mathrm{CA}_{1}$ subregion after global ischemia and reperfusion. The ratio was significantly ameliorated in Tg rats (closed bar) compared with non-Tg littermates (open bar) at $3 \mathrm{~d}(n=6 ; p<0.03)$. However, the ratio was the same between $\mathrm{Tg}$ and non-Tg rats at $1 \mathrm{~d}(n=$ 4). Values are mean \pm SE (Fisher's protected least significant difference test followed by nonparametric $t$ test). E, Quantitative analysis of TUNEL-positive cells in the hippocampal $\mathrm{CA}_{1}$ subregion in SOD1 $\mathrm{Tg}$ rats and non-Tg littermates after transient global cerebral ischemia. TUNEL-positive cells in the hippocampal $\mathrm{CA}_{1}$ subregion were counted by imaging analysis. There was a significant reduction in these DNAfragmented cells in SOD1 Tg rats as compared with non-Tg littermates at $3 \mathrm{~d}$ after $10 \mathrm{~min}$ of ischemia. ${ }^{*} p<0.03$, Fisher's protected least significant difference test followed by nonparametric $t$ test.

Additional Southern blots and reverse transcriptase PCRs have been used during the breeding to confirm both the SOD1 gene and the mRNA, respectively (data not shown). There were no observable phenotypic differences between the SOD1 Tg rats and the littermates.

\section{Reduction of neuronal death and DNA fragmentation in vulnerable hippocampal $\mathrm{CA}_{1}$ neurons after transient global ischemia}

When non-Tg rats were subjected to $10 \mathrm{~min}$ ischemia they developed selective neuronal damage at $3 \mathrm{~d}$ in vulnerable regions, including the hippocampal $\mathrm{CA}_{1}$ subregion, cerebral cortex, thalamus, and striatum, whereas the neurons in the dentate gyrus were not affected (Fig. $6 A$ ). The hippocampal $\mathrm{CA}_{1}$ subregion was the most vulnerable region, and $90 \%$ of the $\mathrm{CA}_{1}$ neurons were lost (Fig. 6A,B). In contrast, neuronal damage in various brain regions was significantly reduced in SOD1 Tg rats (Fig. $7 A, B)$ at $3 \mathrm{~d}$ after reperfusion. The reduction of cell damage in the $\mathrm{CA}_{1}$ region was $\sim 50 \%$ (Fig. $6 B$ ) in $\mathrm{Tg}$ rats. Although there was no significant cell damage in the $\mathrm{CA}_{1}$ subregion at $1 \mathrm{~d}$ after reperfusion in either SOD1 Tg rats or their littermates (Fig. 6B), the significant increase at $3 \mathrm{~d}$ in the non- $\mathrm{Tg}$ animals suggests that a delayed neurodegeneration occurs in the vulnerable $\mathrm{CA}_{1}$ neurons and that increased endogenous SOD1 activity can significantly prevent cell death. Although a neuroprotective effect has been observed in vulnerable neurons of SOD1 $\mathrm{Tg}$ rats after transient global cerebral ischemia, it is not clear whether this neuroprotection is permanent. Such issues can be addressed by future studies of long-delayed cell injury and recovery after ischemia (i.e., 6 months).

The mechanisms of oxidative stress-induced delayed death of hippocampal $\mathrm{CA}_{1}$ pyramidal cells after transient global cerebral ischemia are unclear. Recent studies, although still somewhat controversial, have identified some apoptotic features by biochemical and morphological evidence such as TUNEL (MacManus et al., 1993; Sei et al., 1994; Petito et al., 1997) and internucleosomal DNA fragmentation as indicated by the DNA laddering pattern (Héron et al., 1993). Because TUNEL staining indicates DNA damage, and its specificity for apoptosis is questionable, we have used TUNEL staining only as an indication of DNA damage in cells. Whereas only a small fraction of cells $(<5 \%)$ are TUNEL-positive $1 \mathrm{~d}$ after reperfusion, we have demonstrated a tremendously delayed increase in TUNELpositive cells in the hippocampal $\mathrm{CA}_{1}$ subregion in wild-type animals $3 \mathrm{~d}$ after reperfusion (Fig. $7 E$ ) with up to $40 \%$ of the total cells being TUNEL-positive. The TUNEL-positive cells are likely caused by the increased level of superoxide radicals during reperfusion because $<20 \%$ of the hippocampal $\mathrm{CA}_{1}$ cells are TUNEL-positive in SOD1 Tg rats at $3 \mathrm{~d}$ after reperfusion. Our data also suggest that if TUNEL-positive cells are mainly apoptotic in nature, the damage to hippocampal $\mathrm{CA}_{1}$ neurons would involve both necrosis $(60 \%)$ and apoptosis. However, much more stringent criteria for apoptosis (i.e., DNA laddering, caspases induction, cytochrome c release, and ultrastructural features) will be needed in future studies so that the apoptosis process can be confirmed. Whatever cell death processes are involved in the delayed death of hippocampal $\mathrm{CA}_{1}$ neurons, it is mediated by superoxide radicals. Because only a $50 \%$ reduction in cell death is achieved in SOD1 Tg rats after transient global cerebral ischemia, mechanisms or factors other than superoxide radicals are likely to be involved. It is also noteworthy that superoxide production in the hippocampal $\mathrm{CA}_{1}$ subregion is well developed $24 \mathrm{hr}$ after ischemia, at a time when neuronal damage is not yet observed. This might suggest that superoxide radicals do not immediately damage these neurons but that an interval of time is required for the full expression of the injury. This delayed cell injury might provide a window of opportunity for therapeutic interventions using antioxidants. Additional therapeutic or pharmacological 
regimens in SOD1 $\mathrm{Tg}$ rats will be useful to further dissect the mechanisms involved in delayed vulnerable cell death after transient global cerebral ischemia.

Our success in making SOD1 $\mathrm{Tg}$ rats also provides an impetus for stroke researchers and neuroscientists to develop and to employ these animals for studying the oxidative mechanisms in acute brain injuries and chronic neurodegenerative diseases. Some of these studies are currently being undertaken in our laboratory.

\section{REFERENCES}

Abe K, Aoki M, Kawagoe J, Yoshida T, Hattori A, Kogure K, Itoyama Y (1995) Ischemic delayed neuronal death. A mitochondrial hypothesis. Stroke 26:1478-1489.

Bindokas VP, Jordán J, Lee CC, Miller RJ (1996) Superoxide production in rat hippocampal neurons: selective imaging with hydroethidine. J Neurosci 16:1324-1336.

Boveris A, Chance B (1973) The mitochondrial generation of hydrogen peroxide. Biochem J 134:707-716.

Chan PH (1994) Oxygen radicals in focal cerebral ischemia. Brain Pathol 4:59-65.

Chan PH (1996) Role of oxidants in ischemic brain damage. Stroke 27:1124-1129.

Chan PH, Chu L, Chen SF, Carlson EJ, Epstein CJ (1990) Reduced neurotoxicity in transgenic mice overexpressing human copper-zinc superoxide dismutase. Stroke 21:III80-III82.

Chan PH, Yang GY, Chen SF, Carlson E, Epstein CJ (1991) Posttraumatic brain injury and edema are reduced in transgenic mice overexpressing CuZn-superoxide dismutase. Ann Neurol 21:482-486.

Chan PH, Kinouchi H, Epstein CJ, Carlson E, Chen SF, Imaizumi S, Yang GY (1993) Role of superoxide dismutase in ischemic brain injury: reduction of edema and infarction in transgenic mice following focal cerebral ischemia. Prog Brain Res 96:97-104.

Chan PH, Epstein CJ, Kinouchi H, Kamii H, Chen SF, Carlson E, Gafni J, Yang G, Reola L (1996) Neuroprotective role of CuZn-superoxide dismutase in ischemic brain damage. Adv Neurol 71:271-280.

Copin J-C, Reola LF, Chan TYY, Li Y, Epstein CJ, Chan PH (1996) Oxygen deprivation but not a combination of oxygen, glucose, and serum deprivation induces DNA degradation in mouse cortical neurons in vitro: attenuation by transgenic overexpression of $\mathrm{CuZn}$-superoxide dismutase. J Neurotrauma 13:233-243.

Coyle JT, Puttfarcken P (1993) Oxidative stress, glutamate, and neurodegenerative disorders. Science 262:689-695.

Dugan LL, Sensi SL, Canzoniero LMT, Handran SD, Rothman SM, Lin T-S, Goldberg MP, Choi DW (1995) Mitochondrial production of reactive oxygen species in cortical neurons following exposure to $N$-methyl-D-aspartate. J Neurosci 15:6377-6388.

Epstein CJ, Avraham KB, Lovett M, Smith S, Elroy-Stein O, Rotman G, Bry C, Groner Y (1987) Transgenic mice with increased Cu/Znsuperoxide dismutase activity: animal model of dosage effects in Down syndrome. Proc Natl Acad Sci USA 84:8044-8048.

Héron A, Pollard H, Dessi F, Moreau J, Lasbennes F, Ben-Ari Y, Charriaut-Marlangue C (1993) Regional variability in DNA fragmentation after global ischemia evidenced by combined histological and gel electrophoresis observations in the rat brain. J Neurochem 61: 1973-1976.

Ito U, Kirino T, Kuroiwa T, Klatzo I (1997) Maturation phenomenon in cerebral ischemia II. Neuronal recovery and plasticity. Berlin: Springer.

Kinouchi H, Epstein CJ, Mizui T, Carlson EJ, Chen SF, Chan PH (1991) Attenuation of focal cerebral ischemic injury in transgenic mice over- expressing $\mathrm{CuZn}$ superoxide dismutase. Proc Natl Acad Sci USA 88:11158-11162.

Kirino T (1982) Delayed neuronal death in the gerbil hippocampus following ischemia. Brain Res 239:57-69.

Kondo T, Li Y, Sato S, Murakami K, Copin J-C, Huang T-T, Epstein CJ, Chan PH (1997a) Subcellular localization of superoxide anions following focal cerebral ischemia and reperfusion. J Cereb Blood Flow Metab [Suppl 1] 17:S102.

Kondo T, Reaume AG, Huang T-T, Carlson E, Murakami K, Chen SF, Hoffman EK, Scott RW, Epstein CJ, Chan PH (1997b) Reduction of $\mathrm{CuZn}$-superoxide dismutase activity exacerbates neuronal cell injury and edema formation after transient focal cerebral ischemia. J Neurosci $17: 4180-4189$.

Kontos HA (1985) George E. Brown memorial lecture: oxygen radicals in cerebral vascular injury. Circ Res 57:508-516.

MacManus JP, Buchan AM, Hill IE, Rasquinha I, Preston E (1993) Global ischemia can cause DNA fragmentation indicative of apoptosis in rat brain. Neurosci Lett 164:89-92.

McBean DE, Winters V, Wilson AD, Oswald CB, Alps BJ, Armstrong JM (1995) Neuroprotective efficacy of lifarizine (RS-87476) in a simplified rat survival model of 2 vessel occlusion. Br J Pharmacol 116:3093-3098.

McCord JM (1985) Oxygen-derived free radicals in postischemic tissue injury. N Engl J Med 312:159-163.

Mikawa S, Kinouchi H, Kamii H, Gobbel GT, Chen SF, Carlson E, Epstein CJ, Chan PH (1996) Attenuation of acute and chronic damage following traumatic brain injury in copper, zinc-superoxide dismutase transgenic mice. J Neurosurg 85:885-891.

Murakami K, Kondo T, Epstein CJ, Chan PH (1997) Overexpression of CuZn-superoxide dismutase reduces hippocampal injury after global ischemia in transgenic mice. Stroke 28:1797-1804.

Murakami K, Kondo T, Kawase M, Chan PH (1998a) The development of a new mouse model of global ischemia: focus on the relationships between ischemia duration, anesthesia, cerebral vasculature, and neuronal injury following global ischemia in mice. Brain Res 780:304-310.

Murakami K, Kondo T, Kawase M, Li Y, Sato S, Chen SF, Chan PH (1998b) Mitochondrial susceptibility to oxidative stress exacerbates cerebral infarction that follows permanent focal cerebral ischemia in mutant mice with manganese superoxide dismutase deficiency. J Neurosci 18:205-213.

Petito CK, Torres-Munoz J, Roberts B, Olarte J-P, Nowak Jr TS, Pulsinelli WA (1997) DNA fragmentation follows delayed neuronal death in CA1 neurons exposed to transient global ischemia in the rat. J Cereb Blood Flow Metab 17:967-976.

Pulsinelli WA, Brierley JB, Plum F (1982) Temporal profile of neuronal damage in a model of transient forebrain ischemia. Ann Neurol 11:491-498.

Sei Y, Von Lubitz KJ, Basile AS, Borner MM, Lin RC, Skolnick P, Fossom LH (1994) Internucleosomal DNA fragmentation in gerbil hippocampus following forebrain ischemia. Neurosci Lett 171:179-182.

Shi YP, Huang TT, Carlson EJ, Epstein CJ (1994) The mapping of transgenes by fluorescence in situ hybridization on G-banded mouse chromosomes. Mamm Genome 5:337-341.

Smith ML, Bendek G, Dahlgren N, Rosen I, Wieloch T, Siesjö BK (1984) Models for studying long-term recovery following forebrain ischemia in the rat. 2. A 2-vessel occlusion model. Acta Neurol Scand 69:385-401.

Swanson RA, Morton MT, Wu GT, Savalos R, Davidson C, Sharp FR (1990) A semi-automated method for measuring brain infarct volume. J Cereb Blood Flow Metab 10:290-293.

Yang G, Chan PH, Chen J, Carlson E, Chen SF, Weinstein P, Epstein CJ, Kamii H (1994) Human copper-zinc superoxide dismutase transgenic mice are highly resistant to reperfusion injury after focal cerebral ischemia. Stroke 25:165-170. 